\title{
A descriptive investigation of the ultrastructure of fibrin networks in thrombo-embolic ischemic stroke
}

\author{
Etheresia Pretorius ${ }^{1}{ }^{*}$, Albe C. Swanepoel ${ }^{1}$, Hester M. Oberholzer ${ }^{1}$, Wendy J. van der Spuy ${ }^{1}$, \\ Wiebren Duim ${ }^{2}$, Pieter F. Wessels ${ }^{1}$ \\ ${ }^{1}$ Department of Anatomy, School of Medicine, Faculty of Health Sciences, University of \\ Pretoria, Pretoria, South Africa \\ ${ }^{2}$ Department of Neurology, School of Medicine, Faculty of Health Sciences, University of \\ Pretoria, Pretoria, South Africa
}

*Correspondence to: e-mail: resia.pretorius@up.ac.za

\begin{abstract}
Stroke is one of the leading causes of death worldwide. Formation of a fibrin clot is controlled by a group of tightly regulated plasma proteases and cofactors and a change in the fibrin fiber formation causes an alteration in clot morphology. This plays an important role during thrombotic events. In the current study we investigated the ultrastructure of fibrin networks from fifteen ischemic stroke patients by using scanning electron microscopy. Clot morphology was investigated with and without the addition of human thrombin to the platelet rich plasma. Previously it was shown that, when studying the ultrastructure of fibrin networks, the addition of thrombin is necessary to form an expansive, fully coagulated layer of fibers. Results from the addition of thrombin to the plasma showed thick, matted fibrin fibers and a net covering some of the major fibers in stroke patients. Typical control morphology with major thick fibers and minor thin fibers could be seen in some areas in the stroke patients. In stroke patients, without the addition of thrombin, a matted fibrin network still formed, indicating that the factors responsible for the abnormal fibrin morphology are present in the circulating plasma and is the cause of the observed matted, layered morphology. This is not present in healthy individuals. From the results obtained we suggest that this changed morphology might be useful in a screening regime to identify the possibility of a stroke or even to follow the progress of stroke patients after treatment.
\end{abstract}

Keywords: Thrombo-embolic ischemic stroke; Fibrin networks; Electron microscopy 


\section{Introduction}

Acute stroke is the one of the leading factors of morbidity and mortality worldwide, and is the number three cause of death in the industrialized countries. Approximately 795,000 Americans suffer a new or recurrent stroke each year [1]. Furthermore, in America only, stroke kills more than 137,000 people a year, and it is estimated that Americans will pay about $\$ 73.7$ billion in 2010 for stroke-related medical costs and disability. Stroke is also classified as one of the most important cause of morbidity and long-term disability in Europe and it imposes an enormous economic burden here as well [2].

Ischemic stroke accounts for about $87 \%$ of all stroke cases and approximately $80 \%$ of embolism-related deaths are from stroke and $20 \%$ from other systemic thrombo-embolism [3]. Blood flow is reduced or totally restricted in ischemic stroke that results in neurons being denied of important nutrients. Since glucose is not stored in the brain, and brain metabolism cannot occur in an anaerobic setting, the consequences of ischemia are almost immediate. Ischemia initiated the release of destructive biochemical substances that contribute to tissue destruction. The vascular areas affected by an occlusion will influence the symptoms that will be exhibited. Many ischemic strokes occur without a well-defined aetiology and are labelled as cryptogenic stroke [4]. Altered fibrin clot structure and resistance to fibrinolysis is associated with cryptogenic stroke [5], as well as other ischemic strokes.

Changed clot morphology plays a very important role in thrombotic events, and altered clot morphology is due to changed fibrin fiber formation. Formation of a fibrin clot is controlled by a group of tightly regulated plasma proteases and cofactors [6]. While this system plays an important role in controlling blood loss, it also contributes to pathologic fibrin formation and platelet activation that may occlude vessels [6]. According to Muszbek and co-workers (2008), haemostatic clot formation entails thrombin-mediated cleavage of fibrinogen to fibrin, and thrombin concentration present during clot formation dictates the ultimate fibrin structure. Also, abnormal thrombin generation patterns produce abnormally structured clots that are associated with an increased risk of bleeding or thrombosis [7].

Successful fibrinolysis of the clot plays an important role and is due to the combination of regulated enzymatic activity and the physical properties of the fibrin scaffold [8]. However, Weisel and Litvinov (2008) mentioned that fibrin clots can have a great diversity of structural, biological, physical, and chemical properties depending on the conditions of formation, and the rate and nature of fibrinolysis is related to these properties [8].

The structure of the fibrin clot, which can be characterized in terms of a branched network, directly affects the clot's fibrinolytic and viscoelastic properties, which are remarkable and unique among polymers [9].

\section{Aim}

The aim of the current study is to investigate the ultrastructural characteristics of fibrin networks in stroke patients, by studying the fibrin network using a scanning electron 
microscope. Because research has suggested that the packaging of the fibrin network may be affected in stroke, we aim to investigate if this may be visible.

\section{Methods}

\section{Equipment used}

A Zeiss ULTRA plus FEG-SEM with InLens capabilities were used to study surface morphology and micrographs were taken $1 \mathrm{kV}$. This instrument is located in the Microscopy and Microanalysis Unit of the University of Pretoria, Pretoria, South Africa.

\section{Patients and diagnosis}

Six controls under 40 and six controls over 40 were used in this study and their morphology is in accordance with control samples in previous research. They were free of history of any neurological disease, do not smoke and are not on hormonal therapy. Citrate blood samples were collected within $48 \mathrm{~h}$ post-stroke, from 15 thrombo-embolic ischemic stroke patients (Ethical clearance was obtained from the Research Ethics Committee, Faculty Health Sciences, University of Pretoria, who complies with ICH-GCP guidelines and has US Federal wide Assurance). Exclusion criteria were acute illness, cancer, hepatic or renal dysfunction and the diagnosis was according to the WHO criteria and done by a Neurologist according to the TOAST criteria. This system was developed as a system for categorization of subtypes of ischemic stroke mainly based on aetiology which has been developed for the trial of org 10172 in acute stroke treatment (TOAST) [10]. All patients underwent magnetic resonance (MR) brain scanning (which confirmed the ischemic stroke and excluded all other causes) and standard thrombophilia screening, including (Prothrombin time; Prothrombin Index, Int.Norm. Ratio; PTT; D-Dimers determination and platelet count), were also performed. Although this thrombophilia screening information is not used in the typical diagnosis of stroke, it gives useful information regarding the blood profiles of the patients. A standard stroke work-up including cardiac and carotid ultrasonography, ECG, chest x-ray and blood tests were done. Data concerning demographics and general health status were collected using a questionnaire and smoking was defined as smoking at least five cigarettes per day.

\section{Preparation of fibrin clots}

\section{Citrated whole blood with the addition of thrombin}

Fresh platelet-rich plasma (PRP) from all thrombotic stroke patients was prepared by centrifuging citrated blood at 1,000 rpm (maximum RCF $=17.523 \times \mathrm{g} ; 1250 \mathrm{~g}$ ) for two minutes. The rpm and centrifuge time was the same for controls and patients. Previously, Högman and co-workers in 1982 mentioned that no significant difference of platelet yield in the platelet rich plasma was observed between low g-force for a long time as compared to high g-force for a short time [11]. Centrifugation was performed at room temperature. Human thrombin (provided by the South African National Blood Service) was used to prepare these fibrin clots from the donor. The thrombin solution (which was at room 
temperature) was at a concentration of $20 \mathrm{U} / \mathrm{ml}$ and was made up in a biological buffer containing $0.2 \%$ human serum albumin. When thrombin is added to PRP, fibrinogen is converted to fibrin and intracellular platelet components, e.g. transforming growth factor, platelet-derived growth factor and fibroblastic growth factor are released into the coagulum. $10 \mu \mathrm{l}$ of the PRP was mixed with $10 \mu \mathrm{l}$ of human thrombin on a $0.2 \mu \mathrm{m}$ Millipore membrane to form the coagulum (fibrin clot) (room temperature). This is a standard preparation method to prepare fibrin clots for ultrastructural analysis [12]. The Millipore membrane was placed in a Petri dish on filter paper dampened with phosphate buffered saline (PBS) to create a humid environment and placed at $37^{\circ} \mathrm{C}$ for $10 \mathrm{~min}$ to allow for reaction time.

\section{Citrated whole blood without the addition of thrombin}

$20 \mu \mathrm{l}$ of this PRP was placed on a $0.2 \mu \mathrm{m}$ Millipore membrane. The Millipore membrane was placed in a petri dish on filter paper dampened with phosphate buffered saline (PBS) to create a humid environment and placed at $37^{\circ} \mathrm{C}$ for $10 \mathrm{~min}$. This was followed by a washing process where the Millipore membranes were placed in PBS and magnetically stirred for $20 \mathrm{~min}$. This was done to remove any blood proteins trapped within the fibrin network [12].

\section{Preparation of washed fibrin clots for SEM}

Washed fibrin clots were fixed in a $2.5 \%$ glutaraldehyde/formaldehyde in Dulbecco's Phosphate buffered saline (DPBS) solution with a $\mathrm{pH}$ of 7.4 for $30 \mathrm{~min}$. Each clot was rinsed thrice in phosphate buffer for five minutes before being fixed for 30 min with $1 \%$ osmium tetraoxide $\left(\mathrm{OsO}_{4}\right.$.) The samples were rinsed thrice with $\mathrm{PBS}$ for five minutes and were dehydrated serially in $30,50,70,90 \%$ and three times with $100 \%$ ethanol. The material was critical point dried, mounted and coated with carbon.

\section{Micrograph analyses methodologies}

In order to prevent biased results, in the current study, four morphologists independent of each other, studied the micrographs and each captured the micrographs, independently of each other. This was done blinded; with only lab numbers assigned to the EM stubs of the controls and the patient samples.

\section{Visualization of platelets in blood smears}

On the day of blood collection form patients, three blood smears were made on glass slides. The slides were stained with Rapid Haematological Stain. The presence of platelets was observed using a Nikon Optiphod transmitted light Microscope (Nikon Instech Co., Kanagawa, Japan).

\section{Results}

Table 1 shows general information regarding ischemic stroke patients, including pathology laboratory results. Two of the patients did not have the laboratory tests; however, all 
patients underwent MR scans that confirmed the diagnosis. The TOAST criterion was used by the Neurologist as main diagnosis tool for stroke (Table 1). Table 1, last row, also shows platelet counts of the patient sample. Platelet counts show no significant differences when compared to standard volumes which are $140-450 \times 10^{9} / \mathrm{l}$. The mean platelet count for the patient sample is $2,345 \times 10^{9} /$. This is well within the average platelet count of controls $\left(140-450 \times 10^{9} / \mathrm{I}\right)$. Although literature suggests that the mean platelet volume is significantly higher in patients with ischemic stroke than the control group, Butterworth and Bath in 1998 showed no difference in platelet count between ischemic patients and controls [13]. This might suggest that the circulating factors and their effects on platelets play a more important role than the platelet count.

Table 1. General information of ischemic stroke patients: TOAST criteria and general blood analysis

\begin{tabular}{|c|c|c|c|c|c|c|c|c|c|c|c|c|c|c|c|}
\hline Age & 38 & 44 & 46 & 50 & 55 & 56 & 67 & 71 & 74 & 82 & 84 & 84 & 86 & 62 & 64 \\
\hline Sex & $f$ & $\mathrm{~m}$ & $f$ & $\mathrm{~m}$ & $\mathrm{~m}$ & $\mathrm{~m}$ & $\mathrm{~m}$ & $f$ & $f$ & $f$ & $f$ & $f$ & $f$ & $f$ & $\mathrm{~m}$ \\
\hline Smoke & & & & & & & $x$ & & & & & & & & $x$ \\
\hline Stroke & PACS & PACS & PACS & PACS & PACS & PACS & PACS & PACS & POCS & TACS & PACS & TACS & PACS & PACS & PACS \\
\hline \begin{tabular}{|l} 
Prothrombin \\
time $(10-14 \mathrm{~s})$
\end{tabular} & 11.7 & 12.1 & 12.6 & 11.8 & 11.8 & 12.3 & 8.8 & 13.6 & 19.3 & 11.8 & 11.7 & $\mid 12.4$ & 12.7 & & \\
\hline \begin{tabular}{|l|} 
Prothrombin \\
Index (70-130\%)
\end{tabular} & 106.8 & 113.2 & 98.4 & 105.9 & 117.8 & 100.8 & 108.5 & 90.7| & 67.4 & 111.0 & 118.8 & 104.8 & 102.4 & & \\
\hline \begin{tabular}{|l} 
Int.Norm. Ratio \\
$(0.9-1.2)$
\end{tabular} & 0.9 & 0.9 & 1.0 & 0.9 & 0.8 & 1.0 & 0.92 & 1.1 & 1.6 & 0.9 & 0.8 & 0.9 & 1.0 & & \\
\hline РTT (26-36 s) & 27.1 & 24.8 & $\begin{array}{c}\text { Not } \\
\text { tested }\end{array}$ & 25 & 26.7 & 22.7 & 29.0 & 31.5 & $\begin{array}{c}\text { Not } \\
\text { tested }\end{array}$ & 24 & 24.8 & 29.4 & 25.5 & & \\
\hline \begin{tabular}{|l} 
D-Dimers (0- \\
$0.3 \mathrm{mg} / \mathrm{l})$
\end{tabular} & $\begin{array}{c}\text { Not } \\
\text { tested }\end{array}$ & 1 & $\begin{array}{c}\text { Not } \\
\text { tested }\end{array}$ & $<0.1$ & 0.1 & $<0.1$ & 1.3 & 3.6 & $\begin{array}{c}\text { Not } \\
\text { tested }\end{array}$ & 0.2 & 0.1 & 0.3 & 1.6 & & \\
\hline \begin{tabular}{|l|l} 
Platelet count \\
$\left(140-45010^{9} / \mathrm{I}\right)$
\end{tabular} & 267 & 218 & 240 & 276 & 281 & 288 & 211 & 185 & 247 & 171 & 260 & 238 & 167 & & \\
\hline
\end{tabular}

Numbers in bold represent values higher than average standards for controls

PACS Partial anterior circulation syndrome, TACS Total anterior circulation syndrome, POCS Posterior circulation syndrome, LACS Lacunar syndrome

The prothrombin time (PT) and its derived measures of prothrombin ratio (PR) and international normalised ratio (INR) are measures of the extrinsic pathway of coagulation and determine the clotting tendency of blood. Partial thromboplastin time (PTT) is a blood test that measures the time it takes blood to clot. D-Dimers are breakdown products of fibrin and only present in the presence of clots. At least one of the parameters was present in most of the current stroke patients. Blood was collected from all patients, $48 \mathrm{~h}$ after stroke. Interestingly, no single blood result could be identified and isolated as being responsible for the stroke events in the sample. Different areas in the coagulation cascade, according to the blood results, were changed (Table 1). Because blood was collected $48 \mathrm{~h}$ after stroke in all patients, differences in the profiles cannot be attributed to time frame between onset of clinical symptoms and time of blood analysis. However, these laboratory results, indicating that in all patients, there were abnormalities in the coagulation cascade; and this, together with the MR scan results, and TOAST criteria analysis (shown in Table 1), support the presence of stroke. Interestingly, no literature could be found that stroke 
diagnosis is made specifically only looking at coagulation cascade events. Stroke is always diagnosed by MRI and preferably by using the TOAST criteria.

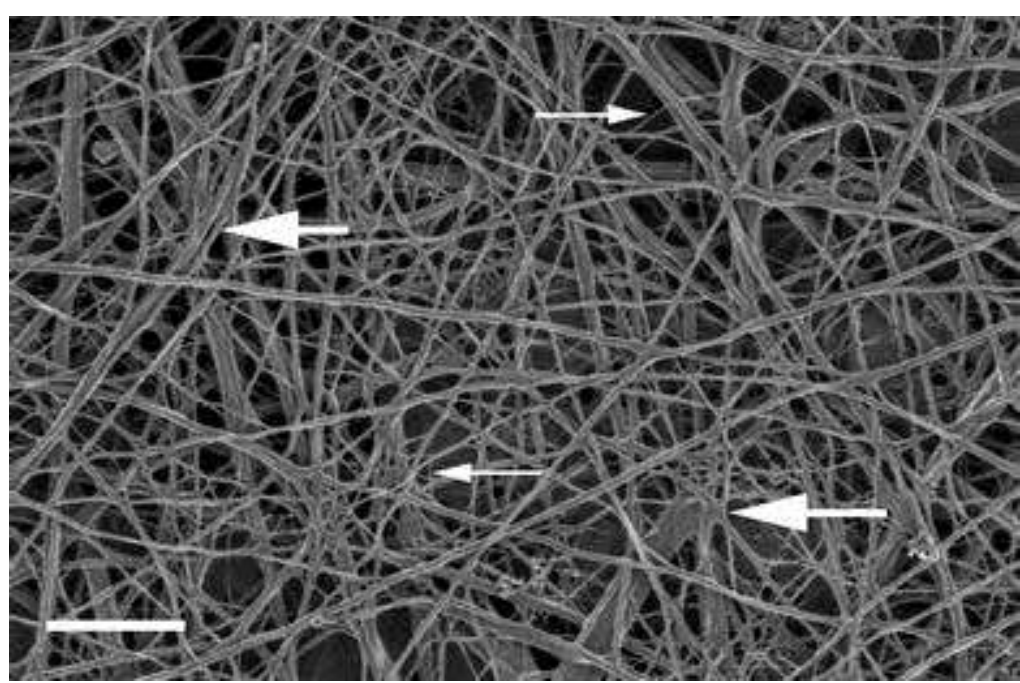

Fig. 1. Control fibrin network created by addition of thrombin to platelet rich plasma. Thick, white arrow major, thick fibers, thin white arrow minor, thin fibers. Scale $1 \mu \mathrm{m}$

Figure 1 shows a typical control fibrin network created with the addition of thrombin; depicting thick, major fibers (thick, white arrow) and thin, minor fibers (thin, white arrow) scattered sparsely in between the major fibers. Figure 2 shows representative micrographs of four of the stroke patients. Thick matted fibrin fibers are visible (Fig. 2, thick white arrows) and a thin net is formed at some areas (Fig. 2, thin, white arrows). Figure 3a shows a micrograph from a patient where fibrin networks were created by adding thrombin and Fig. $3 \mathrm{~b}$ shows the same patient without the addition of thrombin. In Fig. 3a (PRP with additional thrombin) the thick, white arrow shows major, thick fibers and the thin, white arrow, shows netted minor, thin fibers. In Fig. 3b (PRP without thrombin) a platelet is shown with thin, white arrow; and fibrin fibers that are associated with the platelet are shown with thick, white arrows. Fibrin plague is shown with black arrows. 

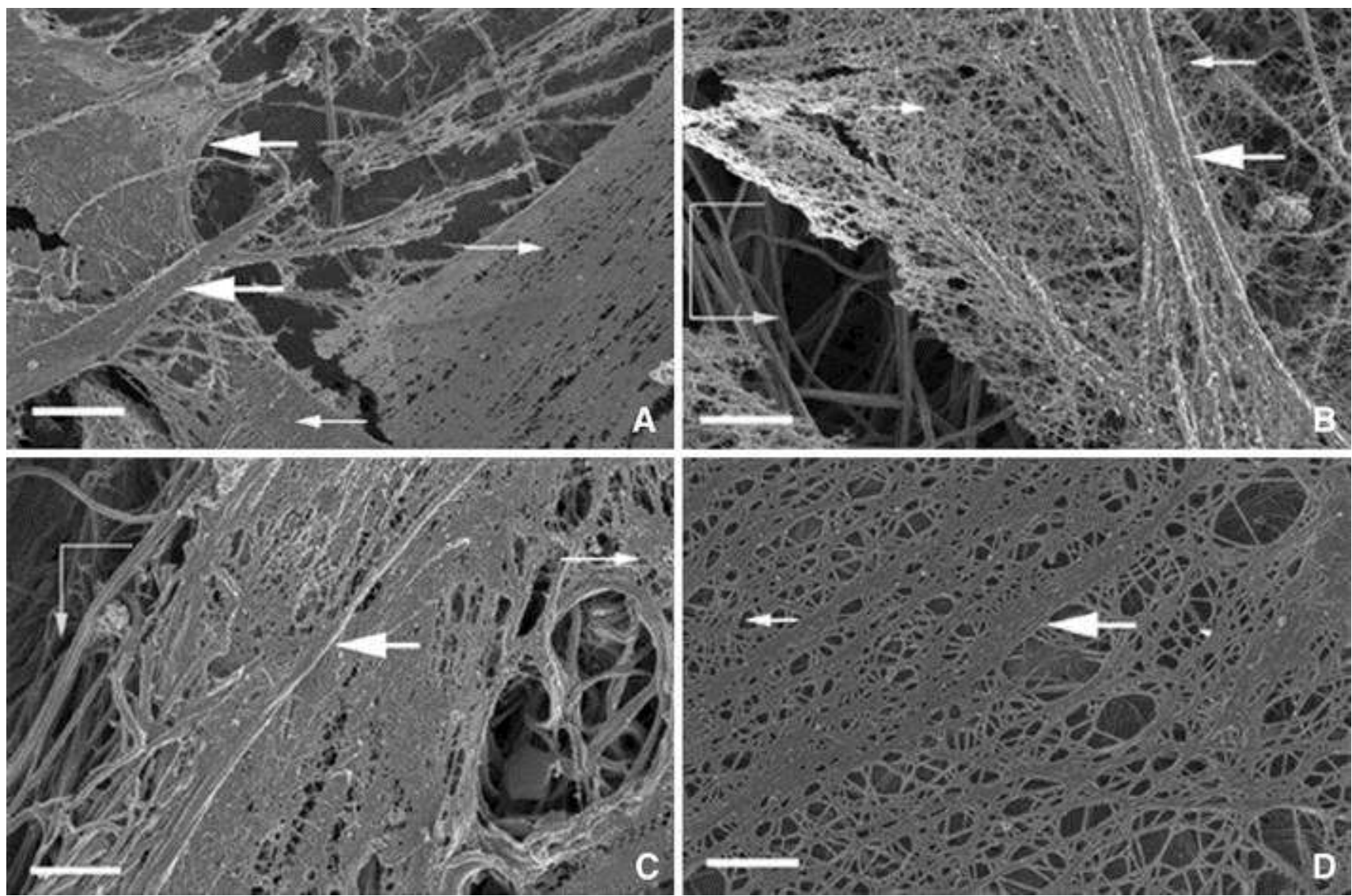

Fig. 2. Fibrin networks from 4 stroke patients created by adding thrombin to platelet rich plasma. Thick, white arrows major, thick fibers, thin white arrow minor, thin fibers, Block arrow small islands of areas of major fibers similar to control fibers. Scale $1 \mu \mathrm{m}$
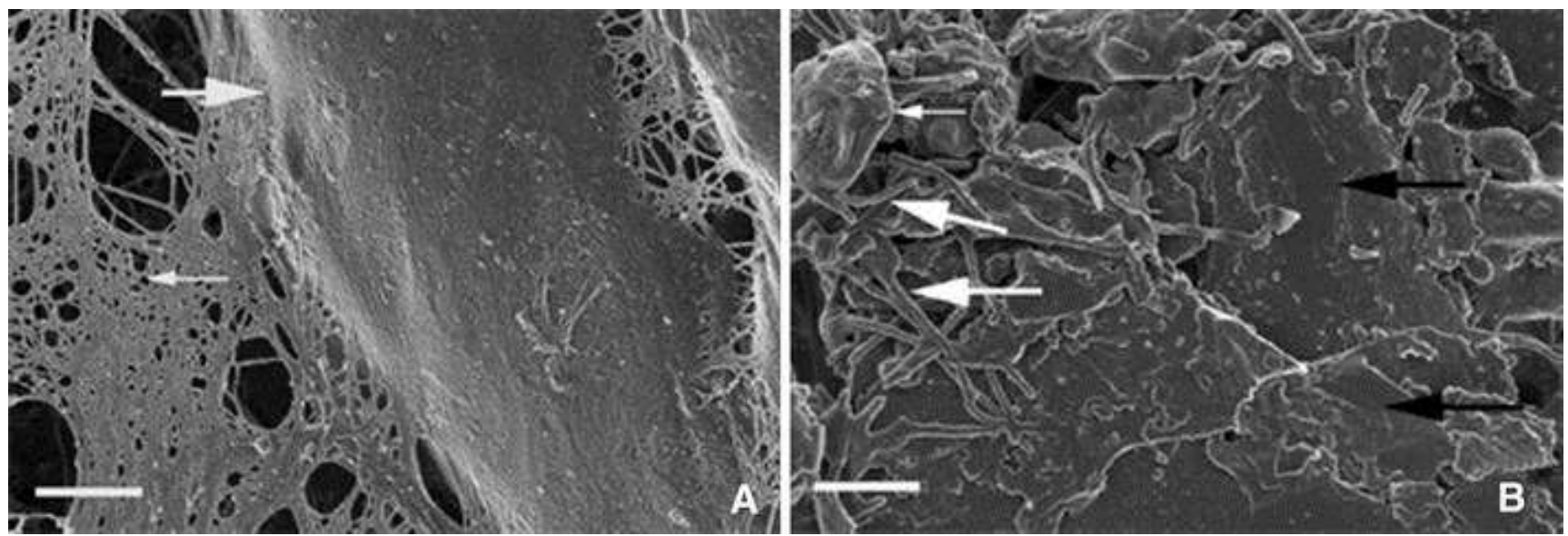

Fig. 3. Comparison of fibrin network created with and without the addition of thrombin to platelet rich plasma from stroke patients. a PRP with additional thrombin; Thick, white arrow major, thick fibers forming densely arranged fibrin plaque, thin, white arrow netted minor, thin fibers. b PRP without thrombin. Thin, white arrow platelet, thick, white arrows fibrin fibers leaving platelet, black arrows fibrin plaques. Scale $1 \mu \mathrm{m}$

\section{Discussion}

Previous research by our group has shown that during inflammation, fibrin morphology changes. It is also well-known that elevated fibrinogen is a cardiovascular risk factor [14] and that abnormal fibrin clot structure, strength and stability correlates with coronary 
artery disease [14]. Recent studies also suggest that patterns of abnormal thrombin generation produce clots with altered fibrin structure and that these changes are associated with an increased risk of bleeding or thrombosis [15]. However, changes in blood coagulation in patients suffering from acute stroke have been described for at least 40 years. Parameters that are well-known, and that confirms this changed coagulation include an activated intrinsic pathway, prothrombin consumption, increased thrombin activity, elevated fibrinogen levels and augmented platelet counts $[16,17]$. Also, increased thrombin activity on platelet surfaces has directly been attributed to fibrin mesh architecture [18].

In the current sample, platelet counts were in the normal range. This agreed with previous research where platelet counts in 30 patients with acute ischemic cerebral infarction were evaluated. Platelet counts were shown to be $207.133 \pm 48.388$ vs. $288.375 \pm 61.373 \times 109 /$ I of controls $(P<0.001)[19]$. The authors suggested that in the earliest phase of stroke, plasma fibrinogen levels may condition the extent of platelet accumulation or consumption in the ischemic area and confirms in vivo that the platelet aggregation process is strictly dependent on fibrinogen concentration [19].

In the current research, we investigated the clot ultrastructure in thrombo-embolic ischemic stroke patients and compare the clot morphology after the addition of thrombin to create clots and clots that formed spontaneously from PRP of stroke patients, without the addition of thrombin. This research in essence confirmed laboratory data from the past 40 years. Table 1 shows general information regarding these patients. All females were postmenopausal and not on hormone replacement therapy. No patient was until the time of stroke, using Warfarin.

Previously, it was shown that, when studying the ultrastructure of fibrin networks, the addition of thrombin is necessary to form an expansive, fully coagulated layer of fibrin fibers in control subjects. Here we investigated whether an expansive fibrin net is present in stroke patients, due to the changed coagulation protein profile from which the stroke resulted.

When comparing the typical control fibrin network with that of the stroke patients, it is noted that in stroke patients, this typical fibrin fiber appearance is changed. Weisel and Litvinov (2008) mentioned that the rate of lysis appears to be faster for clots made up of thicker fibers than for clots made up of thinner fibers [8]. We therefore suggest that this layered, matted fibrin structure that were observed in the stroke patients, may also appear in vivo and because the lysis process take longer, chances are greater for pieces of matted fibrin to cause a thrombotic event.

However, because we are mimicking the creation of a clot by addition of thrombin, we also studied the clot structure of citrate collected PRP, without the addition of thrombin. Previously, it was shown that citrate collected PRP, without the addition of thrombin in controls, produced sparsely arranged thick, major fibrin fibers and only directly associated with platelets. In stroke patients, even without the addition of thrombin, a matted fibrin network forms. The results obtained from the patient with and without the addition of thrombin, as indicated in Fig $3 a$ and $b$, shows that the factors responsible for the abnormal 
fibrin morphology are already present in the plasma and cause a matted, layered morphology. When studying the ultrastructure of fibrin networks in controls, without the addition of thrombin, there is no expansive, fully coagulated layer of fibrin fibers formed. In studying clot morphology, the generation of the clot requires thrombin-mediated conversion of fibrinogen to fibrin [15] and clot formation in vivo occurs in a context of continuous, dynamic changes in thrombin concentration [8]. These changes depend on the local concentrations of pro- and anti-coagulants and cellular activities. Also, in 2010, Undas and co-workers [20] studied the fibrin clot properties in acute ischemic stroke in relation to neurological deficit and concluded that acute stroke is associated with altered fibrin clot properties that might correlate with neurological deficit. They suggested that it remains to be elucidated to what extent these changes have influence on clinical endpoints in patients with this devastating disease. Here, we show how these fibrin networks clot differently from controls and that the ultrastructure is changed to form a thickened, matted morphology, produced mainly by the thin, minor fibers. Also, there are areas that produce plagues of opaque fibrin masses-probably formed by a merging of major fibers.

Ultra-structural analysis in the current sample show strong similarities between fibrin clots generated with or without addition of thrombin. This might suggest high thrombin activity in the PRP of the patients and most likely might be responsible for abnormal ultra-structural findings in the current manuscript. Previous literature support this suggestion, as Wolberg in 2007 [15] mentioned that increased thrombin levels produce thinner fibrin fibers, that are more tightly packed. This is seen in the current research.

\section{Conclusion}

Weisel in 2007 [9] noted that fibrin polymerizes to make clots with a great diversity of structural, biological, physical and chemical properties, depending on the conditions of formation and correlations have been established between these clot properties and many pathophysiological conditions. Here we show that in stroke, fibrin polymerization is changed and this caused a layered, matted fibrin clot structure. Undas and coworkers (2010) [20] showed that there are unfavourably altered fibrin clot properties in stroke and we believe that this changed morphology is probably present long before the actual thrombotic event occurs. We conclude by suggesting that this changed morphology might be used as part of a screening regime to identify the possibility of a stroke or even to follow the progress of stroke patients after treatment. Here, therefore, for the first time, we introduce the thought that SEM might be incorporated in the analysis regime. This has not previously been suggested in the literature, and might have some opposition, especially because the clinical impact of SEM beyond the one of routine clinical chemistry still has to be determined. This might be a feasible option, if a smaller, much more affordable desktop SEM 9 (e.g. a desktop portable SEM manufactured by ZEOL) is used. However, several limitations of this study should be acknowledged, and these include a limited study population and the fact that the analysis was done at a single time point. Also, a more refined patient enrolment protocol may refine the study. We plan a follow-up of these patients after 4 months of the event. This will give additional information about the effectiveness of the treatment regimes on 
clot ultrastructure. Because this study is based on a visual qualitative clot analysis, no statistical analysis is included in the current study.

\section{References}

1. http://www.strokeassociation.org/STROKEORG/AboutStroke/Impact-ofStroke_UCM_310728_Article.jsp (American Heart Association official website: accessed 29 November 2010)

2. http://www.eso-stroke.org/ (European stroke Association-official website: accessed 29 November 2010)

3. Menke J, Lüthje L, Kastrup A, Larsen J (2010) Thromboembolism in atrial fibrillation. Am J Cardiol 105(4):502-510.

4. Schneider AT, Kissela B, Woo D et al (2004) Ischemic stroke subtypes: a population-based study of incidence rates among blacks and whites. Stroke 35:1552-1556.

5. Undas A, Podolec P, Zawilska K et al (2009) Altered fibrin clot structure/function in patients with cryptogenic ischemic stroke. Stroke 40(4):1499-1501.

6. Gailani D, Renné T (2007) Intrinsic pathway of coagulation and arterial thrombosis. Arterioscler Thromb Vasc Biol 27(12):2507-2513.

7. Muszbek L, Bagoly Z, Bereczky Z, Katona E (2008) The involvement of blood coagulation factor XIII in fibrinolysis and thrombosis. Cardiovasc Hematol Agents Med Chem 6(3):190205.

8. Weisel JW, Litvinov RI (2008) The biochemical and physical process of fibrinolysis and effects of clot structure and stability on the lysis rate. Cardiovasc Hematol Agents Med Chem 6(3):161-180.

9. Weisel JW (2007) Structure of fibrin: impact on clot stability. J Thromb Haemost 1:116124.

10. Adams HP Jr, Bendixen BH, Kappelle LJ et al (1993) Classification of subtype of acute ischemic stroke. Definitions for use in a multicenter clinical trial. TOAST. Trial of org 10172 in acute stroke treatment. Stroke 24(1):35-41.

11. Högman CF, Johansson A, Bergius B (1982) A simple method for the standardization of centrifugation procedures in blood component preparation. Vox Sang 43(5):266-269.

12. Pretorius E, Briedenhann S, Marx J et al (2007) Ultra-structural comparison of the morphology of three different platelet and fibrin fiber preparations. Anat Rec 290:188-198. 
13. Butterworth RJ, Bath PM (1998) The relationship between mean platelet volume, stroke subtype and clinical outcome. Platelets 9(6):359-364.

14. Lord ST (2007) Fibrinogen and fibrin: scaffold proteins in hemostasis. Curr Opin Hematol 14(3):236-241.

15. Wolberg AS (2007) Thrombin generation and fibrin clot structure. Blood Rev 21(3):131142.

16. Todd M, McDevitt E, McDowell F (1973) Stroke and blood coagulation. Stroke 4(3):400405.

17. Gaston LW, Brooks JE, Blumenthal HJ, Miller CE (1971) A study of blood coagulation following an acute stroke. Stroke 2(1):81-87.

18. Wolberg AS, Campbell RA (2008) Thrombin generation, fibrin clot formation and hemostasis. Transfus Apher Sci 38(1):15-23.

19. D’Erasmo E, Acca M, Celi FS, Medici F, Palmerini T, Pisani D (1993) Plasma fibrinogen and platelet count in stroke. J Med 24:185-191.

20. Undas A, Slowik A, Wolkow $P$ et al (2010) Fibrin clot properties in acute ischemic stroke: relation to neurological deficit. Thromb Res 125:357-361. 